\title{
Bilateral Mcl Avulsion Following Total knee Arthroplasty: A Case Report and Review of Literature
}

Nishikant Kumar*, Andalib Kashani, John Mukhopadhaya, C.S.Yadav, Sumit Anand, Rajeev Ranjan

Department of Orthopaedics, India

*Corresponding author: Nishikant Kumar, Juan Piazze, Department of Orthopaedics, India

Received date: March 15, 2021 ; Accepted date: March 22, 2021; published date: March 30,2021

Citation: Nishikant Kumar, Andalib Kashani, John Mukhopadhaya , C.S.Yadav, Sumit Anand, et all (2021) Bilateral Mcl Avulsion Following Tka : A Case Report And Review Of Literature J. Clinical Orthopaedics and Trauma Care 3 (1); DOI: 10.31579/2694-0248/011

Copyright: @ 2021 Nishikant Kumar, This is an open access article distributed under the Creative Commons Attribution License, which permits unrestricted use, distribution, and reproduction in any medium, provided the original work is properly cited.

\begin{abstract}
This article describes a traumatic bilateral MCL avulsion in previously well-functioning total knee arthroplasty. A thorough physical examination with appropriate radiographic imaging study is required for proper evaluation and treatment of this injury, as it is associated with debilitating instability of the knee and accelerated wear and failure of the TKA.
\end{abstract}

Keywords: bilateral mcl avulsion; total knee replacement

\section{Introduction}

A traumatic bilateral MCL avulsion after total knee arthroplasty is an extremely rare event, with no case reported in the English literature so far. This report describes a traumatic bilateral MCL avulsion of a previously well-functioning total knee arthroplasty in a 75 year old woman.

\section{Case History}

A 75 year-old female who underwent an uncomplicated sequential bilateral Posterior stabilised total knee replacement presented to the emergency department 15 days post operation with bilateral knee pain and swelling after slipping from stairs.

The patient had severe degenerative osteoarthritis bilateral knee limiting her activities of daily living (Figure 1).

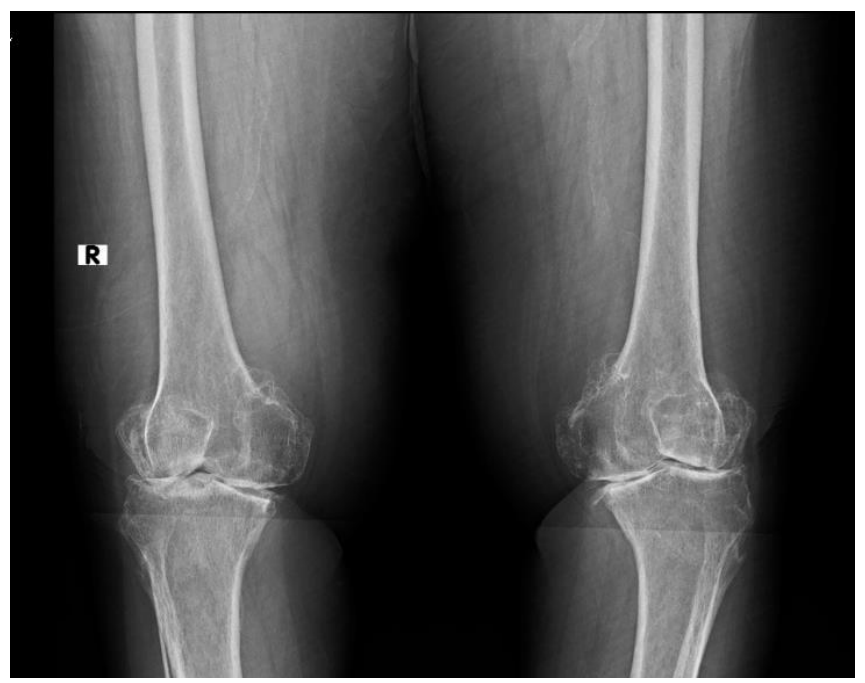

Figure 1: Pre op x ray (Severe OA Bilateral knee)

Sequential TKR (PS Knee) was done with gap of 7 days between the surgeries and it was uneventful. She was mobilised immediately after surgery and regained range of motion up to $110 \mathrm{deg}$. There was no instability demonstrated in her immediate post-op examinations. She started stair climbing 10 days post op. She was discharged $11^{\text {th }}$ day post op of second surgery in stable condition 


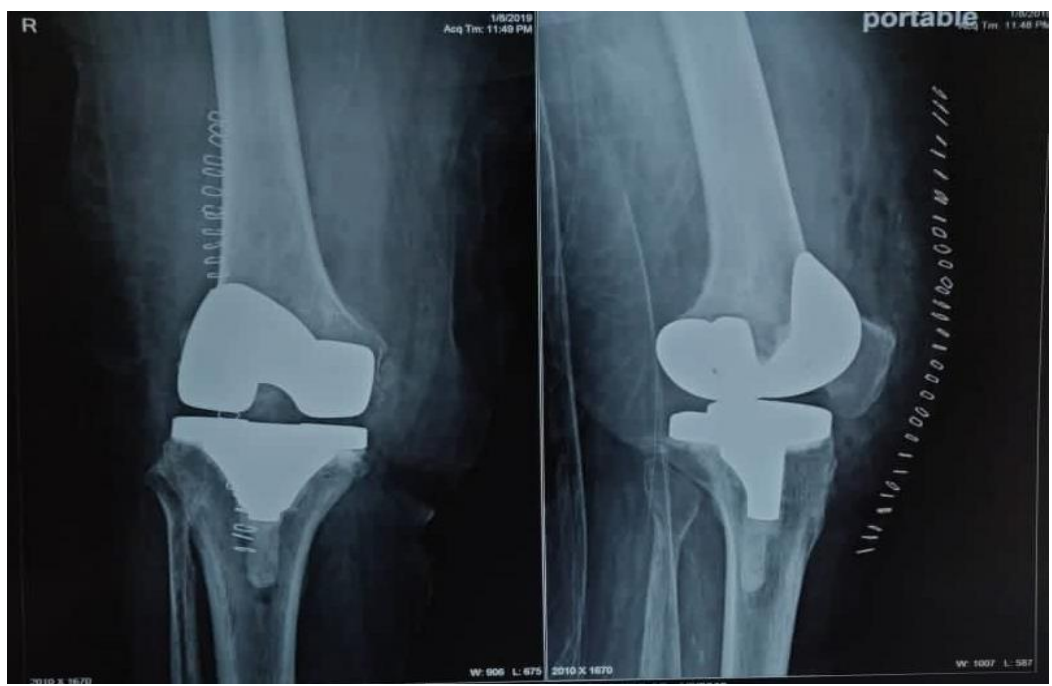

Figure 2: (Post op xray of right Knee)

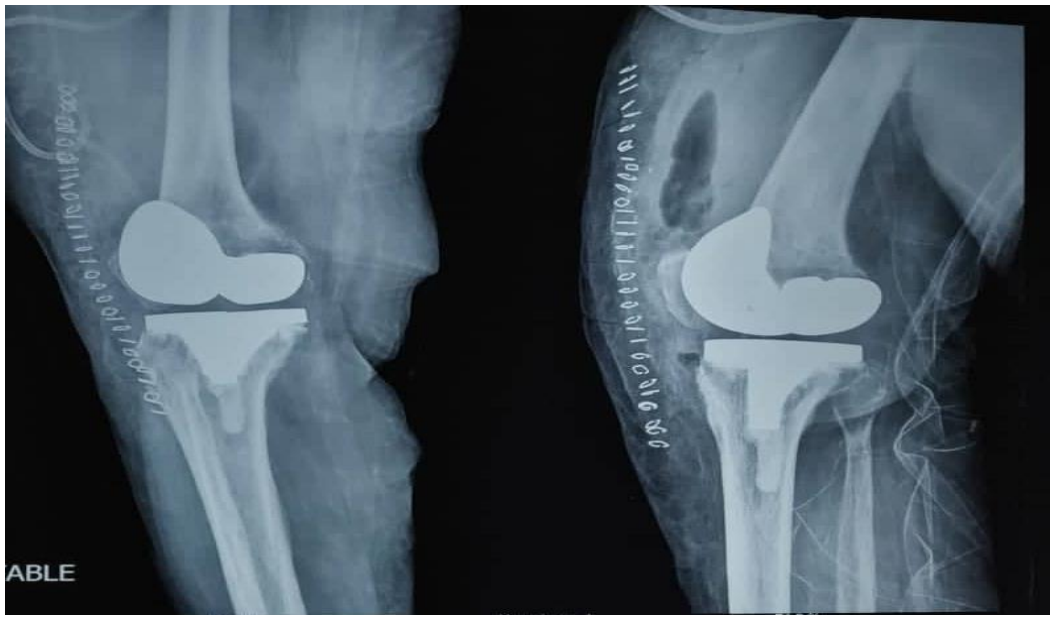

Figure 3: (Post op x-ray Left knee)

She sustained bilateral knee injury on $15^{\text {th }}$ post op of second surgery, when she slipped down the stairs. She immediately experienced pain and swelling in both of her knees and was unable to move her knees afterward. She was unable to ambulate secondary to pain and was brought to the emergency department.

Plain radiographs of bilateral knee AP \& Lat views (Figure 4) and subsequent CT scans with 3D reconstructions demonstrated bilateral MCL avulsion. There was medial condyle fracture with implant loosening over left knee (Type III Lewis and Rorabeck, 1997) while there was MCL Avulsion with stable prosthesis (Type II Lewis and Rorabeck) of the left knee. On clinical examination distal neurovascular status of bilateral lower limbs were found to be intact.

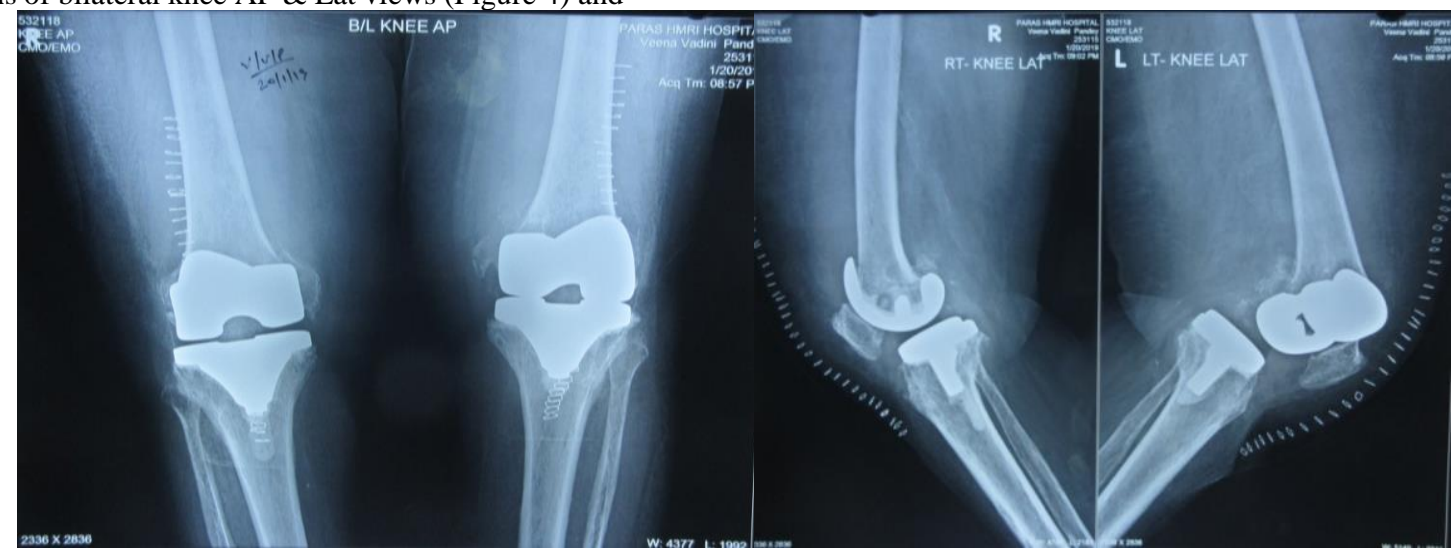

Figure 4: Radiographs of both knees Showing Bilateral MCL Avulsion. 
Patient's both the lower limb were stabilised on above knee slab and all Pre-anaesthetic check-ups were done and patient was posted for surgery after explaining risks to surgery. Both knee were approached sequentially considering the age and medical comorbidity.

Right knee was intervened first. Original skin incision was used, medial parapatellar approach made. Thorough lavage done. Implants were found well fixed. There was bony avulsion of MCL with large chunk of medial femoral condyle. It was stabilised with lag screw and a $\mathrm{T}$-type buttress plate was applied posteromedial. Tibial insert were removed and exchanged with constrained poly. The knee was stable throughout range of motion. Wound was closed in layer with drain inside.
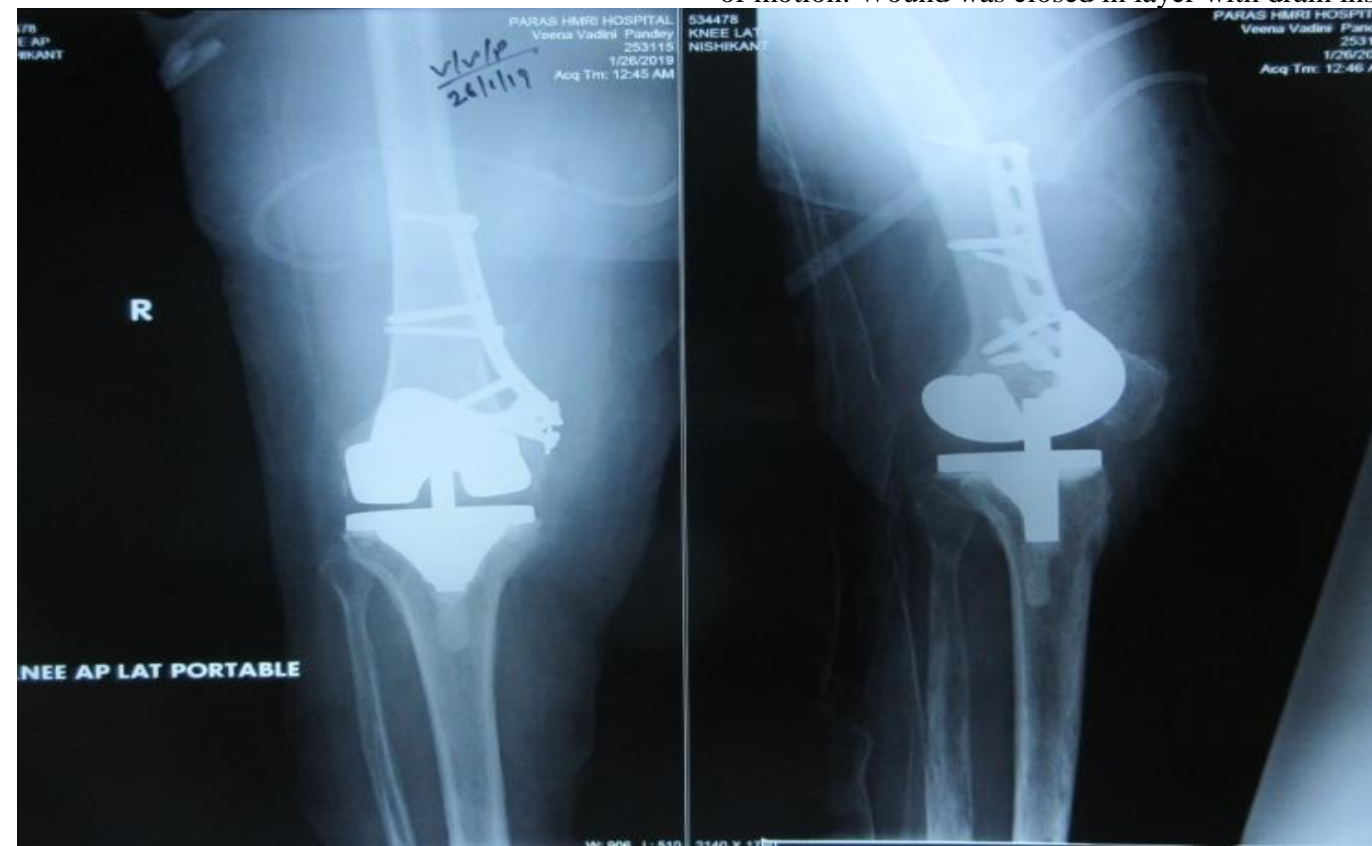

Figure 5: Post op X-ray after Fixing bony avulsion and Poly exchange.

Left knee was approached after a week (for the inflammatory markers to settle). It was also approached through the same midline skin incision and medial parapatellar approach under tourniquet control. The medial epicondyle was found crushed and hardly any bone was left to be fixed. (Figure 6) and femoral component was loose as well. There was no active sign of infection .So it was decided to revise in single stage. Femoral component were taken out. Tibial component was well fixed and it was taken out using saw blade and gentle hammering over osteotomies. It was taken out without taking out any native bone. (Figure 7)

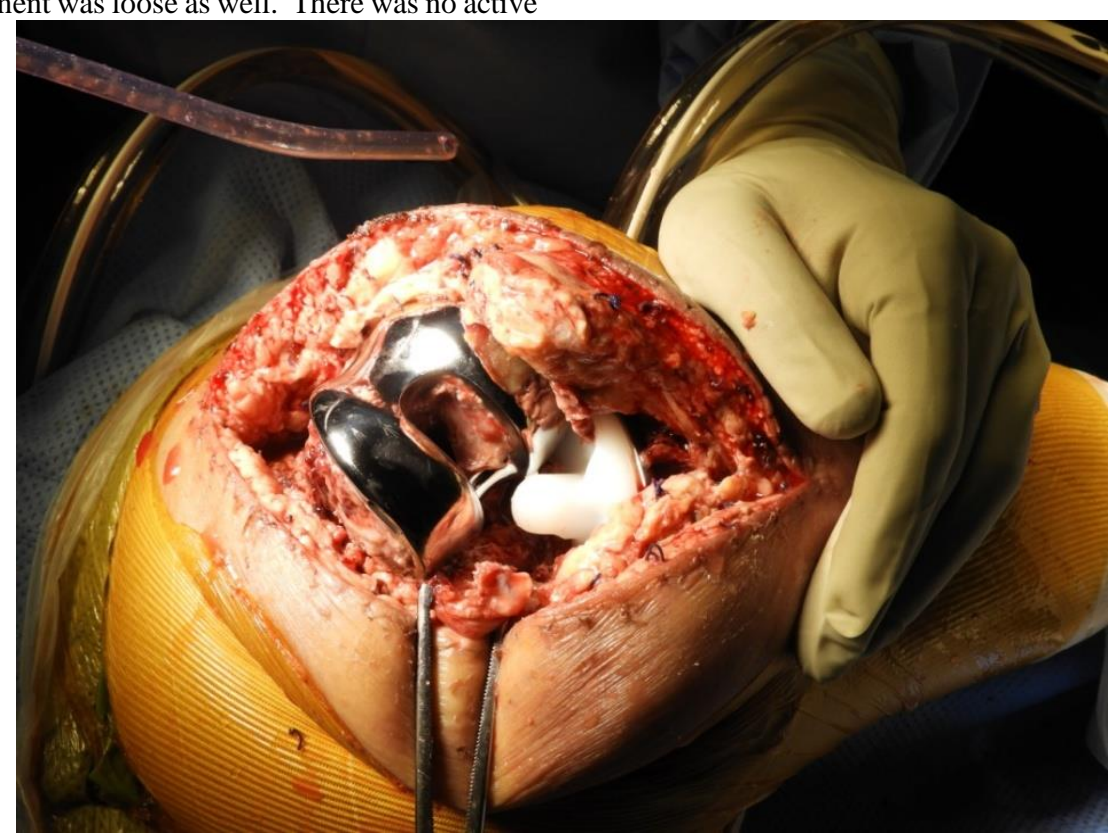

Figure 6: Showing MCL avulsion with Implant in situ. 


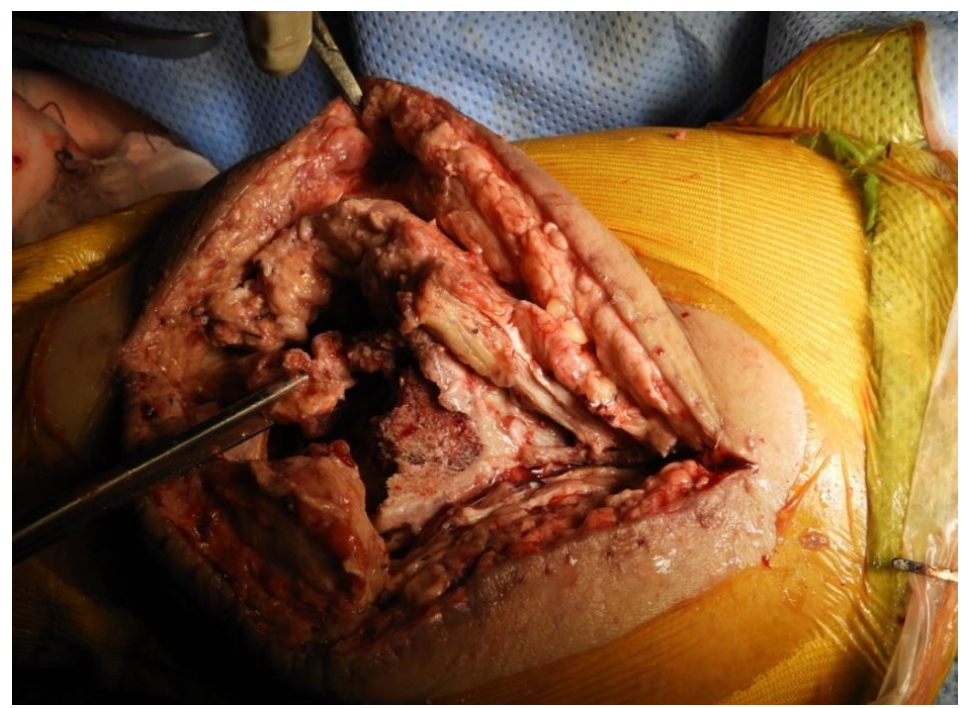

Figurer 7: After Implant Removal.

After thorough lavage Hinge knee (Endomodel; Link) was put in after cementing over both femoral and tibial side. Old meniscal remnant was taken as a guide to ascertain the joint line. Wound was closed in layer with drain insitu.Knee immobilizer was applied.

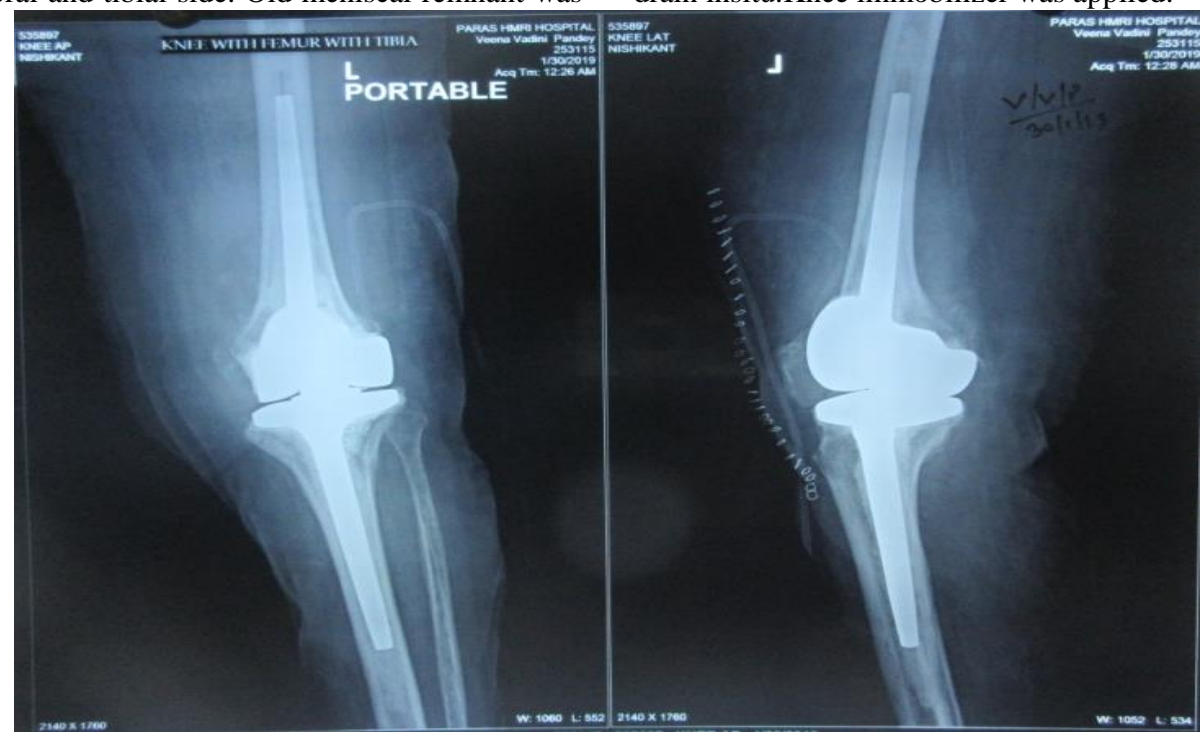

Figure 8: Showing Endomodel hinge knee after revising components.

Patient was given antibiotic prophylaxis for 5 days post op.

The ROM exercises were started next day of surgery on right side while over left side in which fixation was done it was delayed till 2 weeks. Patient was allowed partial weight bearing with walker and Knee immobilizer brace next day of second surgey.The patient's stay in hospital was uneventful, with gradual physiotherapy .Regular wound inspection was done and stiches were removed 2 weeks after second surgery.

Two weeks after her discharge, she was re-examined in the OPD. Swelling and ecchymosis in her both knee had subsided substantially. She kept on using walker and brace for a month and after a month she was advised to use stick for 2 month.

She recovered well in post op period. On subsequent visit at 3 month, 6 month and 1 year follow up she gained 0 to 120 deg movement in both knees without any laxity. She is able to ambulate and climb stairs independently.

\section{Discussion}

A traumatic bilateral MCL avulsion after total knee arthroplasty is an extremely rare event, with no case reported in the English literature so far. Iatrogenic intraoperative disruption of the $\mathrm{MCL}$ is a known complication of TKA seen in about $2.2 \%$ of the patients that can affect patient outcome and satisfaction. [1, 2, 4]

The superficial MCL femoral attachment is approximately $1 \mathrm{~cm}$ anterior and distal to the adductor tubercle and consists of vertical and oblique fibers that coalesce with the anterior tibia approximately $4.5 \mathrm{~cm}$ distal to the medial joint line. The superficial MCL was reported to be the primary restraint to valgus forces by Wymenga et al, [5] who transected the ligament and showed a medial joint line opening of 3 to $5 \mathrm{~mm}$ with valgus stress. The roles of 2 fibre orientations that work in concert with each other were illustrated by Whiteside, [6] who systematically released the anterior fibres first, allowing greater laxity in increasing degrees of flexion, and then the posterior fibers, producing greater laxity in extension. The deep MCL consists of capsular extensions, is typically released during exposure of the posteromedial corner of a varus knee, and 
is considered a secondary restraint to valgus forces. MCL can be injured intraoperatively or postoperatively following subsequent trauma. It can be injured either from femoral side, midsubstance (most common) or from tibial attachment.

Protecting the MCL is an essential aspect of TKA and involves careful placement of retractors and meticulous care in soft tissue handling and bony resections (mostly posterior femoral cut). Morbidly obese and patients with stiff knee are more prone for intraoperative MCL injury. One common situation intraoperatively when MCL gets ruptured is when we try to forcefully negotiate trial insert with less created flexion space. This habit of forceful insertion should be inhibited and we should go on doing soft tissue release or revising bone cuts before putting trial components.

After u recognise MCL injury you first have to recognise the site of injury then it can be either repaired or augment with graft.

Femoral Avulsion- If MCL is avulsed from femoral attachment it can be sutured back using suture anchor or spike washers or staples. [9, 10]

Tibial avulsion- It can again be sutured back using anchors or staples. [4, $10,12]$

Midsubstance tear- Primary repair with mobilisation has shown bad result in various literatures. Some author recommend Primary repair and casting , which is also not very encouraging[4] .Good results have been documented with Primary repair and augmenting this repair with double hamstring graft, keeping tibial attachment of hamstring intact, fixing graft to medial epicondyle and tensioning it at 30 deg flexion. $[12,13]$

Some literature recommended the use of more constrained implants to restore coronal plane stability if the MCL was deemed incompetent or disrupted.[3] However, increasing the level of constraint can result in various concerns that a surgeon must take into consideration before selecting this type of implant. Also increasing level of constraint is not going to give varus and valgus stability. Condylar constrained prosthetic designs increase the stresses on implant fixation, which can play a role in aseptic loosening. [3]. If these methods don't work we may have to revise it using Hinge knee. In the presence of a disrupted MCL, the PCL substituting implant provided no valgus stability in any degree of flexion $[8,12,13]$.

Proper TKA balancing relies on appropriate soft tissue tension provided by intact collateral ligaments. Loss of the medial soft tissue structures such as the MCL can result in instability and accelerated wear, necessitating the need for a revision. Currently, no specific recommendations have been universally agreed on for this situation.

The case discussed in this article is unique as it describes a traumatic bilateral MCL avulsion following TKR after sustaining a fall from stairs. This diagnosis should be considered when presented with a swollen and painful knee with limited range of motion with valgus stress test positive in a patient who previously underwent total knee arthroplasty. Recognition of this injury and proper surgical intervention is imperative for the outcome of TKR.The treatment recommendation is immediate immobilization of the knee joint and a careful physical examination to assess neurovascular status often requiring advanced imaging such as computed tomography.

Based on our experience with this patient, we recommend immediate immobilization and protection of the injured knee followed by surgical exploration. It's important to assess the stability of implant and size of bone attached with MCL. If there is Bony avulsion of MCL which is fixable and implant is not loose then it should be fixed with screw and buttress plate. However if there is implant loosening its advisable to revise it with hinge knee.
Post operatively the limb should be immobilised for at least 3 weeks if repair or fixation has been done. Gentle physiotherapy; ROM exercises with protected delayed weight bearing is advisable subsequently.

\section{Summary}

A bilateral traumatic avulsion of the MCL after a total knee arthroplasty is a very rare injury. Our case report describes a patient who sustained this injury after fall from stairs. This injury can be associated with suboptimal outcome of a TKR. Therefore, it is important to perform a thorough clinical and radiological assessment of the injury and exploration of the joint. Most important is to assess the stability of implant. If implant is stable we can try repair with augmentation or fixation if good amount of bone left. However if implant is loose there is no point retaining it and it should be revised with hinge knee. Also the surgeon needs to be guarded about the prognosis with gentle physiotherapy and protected delayed weight bearing as well as regular clinical and radiological examinations subsequently.

\section{References}

1. Villanueva M, Ríos-Luna A, Pereiro J, Fahandez-Saddi H, PerezCaballer A.Dislocation following total knee arthroplasty: a report of six cases. Indian JOrthop 2010; 44(4):438.

2. Halvorson JJ, Anz A, Langfitt M, et al. Vascular injury associated with extremity trauma: initial diagnosis and management. J Am Accad Orthop Surg 2011; 19(8):495.

3. C.H. Yan, K.Y. Chiu, F.Y. Ng Anterior dislocation of Insall Burstein II total knee arthroplasty secondary to polyethylene tibial post fracture: a case report J Orthop Surg (Hong Kong), 18 (3) (2010), p. 385

4. Lee GC, Lotke PA. Management of intraoperative medial collateral ligament injury during TKA. Clin Orthop Relat Res. 2011; 469(1):64-68.

5. Wymenga AB, Kats JJ, Kooloos J, Hillen B. Surgical anatomy of the medial collateral ligament and the posteromedial capsule of the knee [published online ahead of print October 26, 2005]. Knee Surg Sports Traumatol Arthrosc. 2006; 14(3):229-234.

6. Whiteside LA. Correction of ligament and bone defects in total arthroplasty of the severely valgus knee. Clin Orthop Relat Res. 1993; (288):234-245.

7. Winiarsky R, Barth P, Lotke P. Total knee arthoplasty in morbidly obese patients. J Bone Joint Surg Am. 1998; 80(12):1770-1774.

8. Saeki K, Mihalko WM, Patel V, et al. Stability after medial collateral ligament release in total knee arthoplasty. Clin Orthop Relat Res. 2001; (392):184-189.

9. Leoplod SS, Mcstay C ,Klafeta K,Jacobs JJ; Primary repair of intraoperative disruption of the medical collateral ligament during total knee arthroplasty: J Bone Joint Surg Am. 2001 Jan;83(1):8691.

10. Bohl DD, Wetters NG, Del Gaizo DJ, Jacobs JJ, Rosenberg AG, Della Valle CJ. Repair of Intraoperative Injury to the Medial Collateral Ligament during Primary Total Knee Arthroplasty. J Bone Joint Surg Am. 2016 Jan 6; 98(1):35-39.

11. Dragosloveanu S1, Cristea S, Stoica C, Dragosloveanu C. Outcome of iatrogenic collateral ligaments injuries during total knee arthroplasty. Eur J Orthop Surg Traumatol. 2014 Dec; 24(8):1499-1503.

12. Adravanti P1, Dini F2, Calafiore G1, Rosa MA2. Medial collateral ligament reconstruction during TKA: a new approach and surgical technique. Joints. 2016 Jan 31; 3(4):215-217.

13. Wierer $\mathrm{G}$ et al., Anatomical MCL reconstruction following TKA; Knee 2016.23(5);911-914 